Published in final edited form as:

Eur Urol. 2013 June ; 63(6): 1082-1090. doi:10.1016/j.eururo.2012.06.029.

\title{
Impact of Smoking on Oncologic Outcomes of Upper Tract Urothelial Carcinoma After Radical Nephroureterectomy
}

\author{
Michael Rink $^{a, b}$, Evanguelos Xylinas ${ }^{a, c}$, Vitaly Margulis ${ }^{d}$, Eugene K. Cha ${ }^{a}$, Behfar Ehdaie ${ }^{a}$, \\ Jay D. Raman ${ }^{e}$, Felix K. Chun ${ }^{b}$, Kazumasa Matsumoto ${ }^{f}$, Yair Lotan ${ }^{d}$, Helena Furberg ${ }^{g}$, \\ Marek Babjuk ${ }^{\mathrm{h}}$, Armin Pycha ${ }^{\mathrm{i}}$, Christopher G. Wood', Pierre I. Karakiewicz ${ }^{\mathrm{k}}$, Margit Fisch ${ }^{\mathrm{b}}$, \\ Douglas S. Scherra ${ }^{\mathrm{a}}$, and Shahrokh F. Shariat ${ }^{\mathrm{a}, \mathrm{l},{ }^{*}}$ for the Upper Tract Urothelial Carcinoma \\ Collaboration \\ ${ }^{a}$ Department of Urology, Weill Cornell Medical College, New York-Presbyterian Hospital, New \\ York, NY, USA \\ ${ }^{b}$ Department of Urology, University Medical Center Hamburg-Eppendorf, Hamburg, Germany \\ 'Department of Urology, Cochin Hospital, APHP, Paris 5 University, Paris, France \\ dDepartment of Urology, University of Texas Southwestern Medical Center, Dallas, TX, USA \\ eDepartment of Urology, Penn State Milton S. Hershey Medical Center, Hershey, PA, USA \\ fDepartment of Urology, Kitasato University School of Medicine, Sagamihara, Kanagawa, Japan \\ gDepartment of Epidemiology and Biostatistics, Memorial Sloan-Kettering Cancer Center, New \\ York, NY, USA \\ hDepartment of Urology, Hospital Motol, 2nd Faculty of Medicine, Charles University, Prague, \\ Czech Republic
}

(C) 2012 European Association of Urology. Published by Elsevier B.V. All rights reserved.

*Corresponding author. Department of Urology and Division of Medical Oncology, Weill Medical College of Cornell University, New York-Presbyterian Hospital, 525 East 68th Street, Box 94, Starr 900, New York, NY 10065. Tel.: +1 2127465562.

sfshariat@gmail.com (S. F. Shariat).

Author contributions: Shahrokh F. Shariat had full access to all the data in the study and takes responsibility for the integrity of the data and the accuracy of the data analysis.

Study concept and design: Shariat, Rink.

Acquisition of data: Shariat, Margulis, Raman, Matsumoto, Lotan, Pycha, Wood, Scherr.

Analysis and interpretation of data: Shariat, Rink.

Drafting of the manuscript: Rink, Shariat, Xylinas, Cha.

Critical revision of the manuscript for important intellectual content: Rink, Xylinas, Margulis, Cha, Ehdaie, Raman, Chun,

Matsumoto, Lotan, Furberg, Babjuk, Pycha, Wood, Karakiewicz, Fisch, Scherr, Shariat.

Statistical analysis: Rink, Shariat, Karakiewicz.

Obtaining funding: None.

Administrative, technical, or material support: None.

Supervision: Shariat.

Other (specify): None.

Financial disclosures: Shahrokh F. Shariat certifies that all conflicts of interest, including specific financial interests and relationships and affiliations relevant to the subject matter or materials discussed in the manuscript (eg, employment/ affiliation, grants or funding, consultancies, honoraria, stock ownership or options, expert testimony, royalties, or patents filed, received, or pending), are the following: Michael Rink is a speaker for Pfizer Pharma. He is supported by the Frederick J. and Theresa Dow Wallace Fund of the New York Community Trust. Shahrokh F. Shariat is on the advisory board of Ferring Pharma. The other authors have nothing to disclose.

Publisher's Disclaimer: This is a PDF file of an unedited manuscript that has been accepted for publication. As a service to our customers we are providing this early version of the manuscript. The manuscript will undergo copyediting, typesetting, and review of the resulting proof before it is published in its final citable form. Please note that during the production process errors may be discovered which could affect the content, and all legal disclaimers that apply to the journal pertain. 
'Department of Urology, General Hospital of Bolzano, Bolzano, Italy

iDepartment of Urology, MD Anderson Cancer Center, Houston, TX, USA

kDepartment of Urology, University of Montreal, Montreal, QC, Canada

'Division of Medical Oncology, Weill Cornell Medical College, New York-Presbyterian Hospital, New York, NY, USA

\section{Abstract}

Background-Ci garette smoking is a common risk factor for developing upper tract urothelial carcinoma (UTUC).

Objective-To assess the impact of cigarette smoking status, cumulative smoking exposure, and time from cessation on oncologic UTUC outcomes in patients treated with radical nephroureterectomy (RNU).

Design, setting, and participants-A total of 864 patients underwent RNU at five institutions. The median follow-up in this retrospective study was 50 mo. Smoking history included smoking status, quantity of cigarettes per day (CPD), duration in years, and years from smoking cessation. The cumulative smoking exposure was categorized as light-short-term ( $\leq 9$ $\mathrm{CPD}$ and $\leq 9.9 \mathrm{yr}$ ), moderate (all combinations except light-short-term and heavy-long-term), and heavy-long-term ( $\geq 20 \mathrm{CPD}$ and $\geq 20 \mathrm{yr}$ ).

Interventions-RNU with or without lymph node dissection. No patient received neoadjuvant chemotherapy.

Outcome measurements and statistical analysis-Univariable and multivariable logistic regression and competing risk regression analyses assessed the effects of smoking on oncologic outcomes.

Results and limitations-A total of 244 patients (28.2\%) never smoked; 297 (34.4\%) and 323 (37.4\%) were former and current smokers, respectively. Among smokers, 87 (10.1\%), 331 (38.3\%), and $202(23.4 \%)$ were light-short-term, moderate, and heavy-long-term smokers, respectively. Current smoking status, smoking $\geq 20 \mathrm{CPD} \geq 20 \mathrm{yr}$, and heavy-long-term smoking were associated with advanced disease ( $p$ values $\$$ ).004), greater likelihood of disease recurrence ( $p$ values $\unlhd_{0.01}$ ), and cancer-specific mortality ( $p$ values $\$ 0.05$ ) on multivariable analyses that adjusted for standard features. Patients who quit smoking $\geq 10 \mathrm{yr}$ prior to RNU did not differ from never smokers regarding advanced tumor stages, disease recurrence, and cancer-specific mortality, but they had better oncologic outcomes then current smokers and those patients who quit smoking $<10 \mathrm{yr}$ prior to RNU. The study is limited by its retrospective nature.

Conclusions-Cigarette smoking is significantly associated with advanced disease stages, disease recurrence, and cancer-specific mortality in patients treated with RNU for UTUC. Current smokers and those with a heavy and long-term smoking exposure have the highest risk for poor oncologic outcomes. Smoking cessation $>10$ yr prior to RNU seems to mitigate some detrimental effects. These results underscore the need for smoking cessation and prevention programs.

\section{Keywords}

Smoking; Urothelial carcinoma; Transitional cell carcinoma; Upper urinary tract; Radical nephroureterectomy; Dose-response relationship; Recurrence; Survival; Prognosis 


\section{Introduction}

Smoking is one of the most common risk factors for developing urothelial carcinoma (UC), including upper tract urothelial carcinoma (UTUC) [1]. Smoking increases the relative risk of developing this rare but potentially lethal disease by 2.5 - to 7 -fold [2-4]. One previous study of 502 UTUC patients showed that the risk of developing UTUC increases with an increasing number of daily smoked cigarettes and years of smoking, even when controlling for the effects of age and gender [5]. Smoking cessation $>10 \mathrm{yr}$ earlier seems to decrease the effect of smoking on UTUC development [5].

Radical nephroureterectomy (RNU) with excision of a bladder cuff is the standard of care for patients with high-grade and/or invasive tumors of the renal pelvicalyceal system or ureters with a normal contralateral kidney $[1,6]$. At the time of RNU, up to 50\% of patients have non-organ-confined disease or lymph node metastasis; $50-70 \%$ of these patients eventually die of UTUC within $5 \mathrm{yr}$ of their RNU [6-8]. Cumulative evidence suggests an association between smoking and more advanced disease stage and grade as well as disease recurrence in urothelial carcinoma of the bladder (UCB) [9-13]. However, to the best of our knowledge, no study has yet assessed the impact of different aspects of smoking behavior on UTUC outcomes in patients treated with RNU.

We hypothesized that smoking is associated with biologically more aggressive tumors as reflected by pathologic features and survival outcomes. We also hypothesized that there is a dose-response relationship between smoking intensity and adverse outcomes and that smoking cessation may reduce these effects. To address these hypotheses, we investigated smoking habits and intensity as well as cessation from smoking in a large international multi-institutional cohort of patients treated with RNU for UTUC.

\section{Patients and methods}

\subsection{Patient selection}

This was a retrospective study approved by the institutional review board, with all participating sites providing the necessary data-sharing agreements prior to initiation. A total of five centers provided data. A computerized databank was generated for data transfer. After combining the data sets, reports were generated for each variable to identify data inconsistencies and other data integrity problems. Through regular communication with all sites, resolution of all identified anomalies was achieved before analysis. Prior to final analysis, the database was frozen. The Upper Tract Urothelial Carcinoma Collaboration collected data on 2492 patients with UTUC. Patients with a history of radical cystectomy for treatment of muscle-invasive or high-risk non-muscle-invasive bladder cancer were excluded from data collection. In total, 564 had missing data on smoking status, 927 patients had missing data on smoking quantity, duration, or time from cessation, and 111 patients had missing data on clinicopathologic variables or follow-up and therefore were excluded from the analysis. Patients reporting tobacco use other than cigarette smoking (eg, tobacco chewing, cigars, and pipes) were excluded $(n=26)$. Complete data of 864 consecutive patients who underwent RNU (open: 741 [85.8\%]; laparoscopic: 123 [14.2\%]) between 1987 and 2007 for UTUC were available. No patient received preoperative systemic chemotherapy or perioperative radiotherapy. RNU was performed according to techniques previously described [14]. Hilar or regional lymphadenectomy was generally performed in patients with suspicious lymph nodes on preoperative imaging or with suspicious intraoperative findings [15]. The indication and extent of lymphadenectomy performed was at the discretion of the individual surgeons. Tumor multifocality was defined as the synchronous presence of two or more pathologically confirmed tumors in any location (renal 
pelvicalyceal system or ureter). Adjuvant chemotherapy was administered at the investigator's discretion.

\subsection{Pathologic evaluation}

All surgical specimens were processed according to standard pathologic procedures at each institution. Genitourinary pathologists who were blinded to clinical outcomes reexamined all specimens according to standardized criteria and confirmed UC histology. Tumors were staged according to the 2010 American Joint Committee on Cancer/Union Internationale Contre le Cancer TNM classification [16]. Tumor grading was performed according to the 2004 World Health Organization/International Society of Urologic Pathology consensus classification [17]. Histopathologic assessment included concomitant carcinoma in situ, tumor architecture (papillary or sessile based on the predominant feature of the index lesion [18]), lymphovascular invasion (defined as the presence of tumor cells within an endothelium-lined space without underlying muscular walls [8]), and tumor necrosis (defined as the presence of microscopic coagulative necrosis in $>10 \%$ of the tumor [19]). Tumor location was defined as either renal pelvicalyceal or ureteral based on the index cancer [14].

\subsection{Smoking assessment}

Smoking history was routinely assessed at a clinic visit within $1 \mathrm{yr}$ of RNU. Patients were only considered ever smokers if they had smoked 100 cigarettes during their lifetime. Data on self-reported cigarette smoking included smoking status (current, former, or never smoker), average number of cigarettes per day (CPD; ie, quantity; never smoked, 1-9, 10$19,20-29, \geq 30$ ), duration in years (never smoked, $₫ 9.9,10-19.9,20-29.9,30-39.9, \geq 40$ ), and years since smoking cessation to RNU in former smokers ( $\leq 4.9,5-9.9, \geq 10 \mathrm{yr}$ ). Patients who reported smoking cessation within $1 \mathrm{yr}$ prior to surgery were considered current smokers.

\subsection{Follow-up regimen}

Patients were generally followed every 3-4 mo for the first year following RNU, every 6 mo from the second through the fifth year, and annually thereafter [6]. Follow-up consisted of a history, physical examination, routine blood work, urinary cytology, chest radiography, cystoscopic evaluation of the urinary bladder, and radiographic evaluation of the contralateral upper urinary tract. Elective bone scans, chest computed tomography scans, or magnetic resonance imaging was performed when clinically indicated. Disease recurrence was defined as tumor relapse in the operative field, regional lymph nodes, and/or distant metastasis. Occurrences of UC in the bladder or contralateral upper tract were not considered disease recurrence. Cause of death was determined by treating physicians, by chart review corroborated by death certificates, or by death certificates alone [20]. All patients who were coded as dead of cancer had previous disease recurrence. Perioperative mortality (ie, any death within $30 \mathrm{~d}$ of surgery or before discharge) was censored at the time of death for cancer-specific survival analyses.

\subsection{Statistical analysis}

For statistical analyses, smoking quantity (never vs $\leq 19$ vs $\geq 20$ CPD), duration (never vs $\leq 9$ vs $\geq 20 \mathrm{yr}$ ), and years since cessation (never vs $₫ 9.9 \mathrm{vs} \geq 10 \mathrm{yr}$ vs current smoking) were grouped based on previous findings [21-23] and preliminary analyses (supplementary Fig. $1 \mathrm{a}$ and $1 \mathrm{~b}$ ). We categorized patients based on their cumulative smoking exposure into four groups: never smoker, light-short-term smoker ( $\triangle 9 \mathrm{CPD}$ and $\leq 19.9 \mathrm{yr}$ ), moderate smoker ( $\geq 20 \mathrm{CPD}$ and $₫ 9.9 \mathrm{yr}$ or $₫ 9 \mathrm{CPD}$ and $\geq 20 \mathrm{yr}$ ), and heavy-long-term smokers ( $\geq 20 \mathrm{CPD}$ 
and $\geq 20 \mathrm{yr}$ ). Preliminary analyses using different cut-offs revealed this stratification to offer the best discrimination for cumulative smoking exposure.

The Kolmogorov-Smirnov test was used to assess the distribution of variables. The Fisher exact test and the chi-square test were used to evaluate the association between categorical variables. Differences in variables with a continuous distribution across categories were assessed using the Mann-Whitney $U$ test (two categories) and Kruskal-Wallis test (three and more categories). Using multivariable logistic regression analyses, the association of smoking with pathologic stage and lymph node metastasis at the time of RNU was assessed in two models (end points: prediction of $\ngtr \mathrm{pT} 2$ and/or $\mathrm{pN}+, \mathrm{pT}^{\mathrm{T}} 3$, and/or $\mathrm{pN}+$ ). Each model adjusted for the effects of age, gender, and tumor location. To assess the impact of smoking on disease recurrence and cancer-specific mortality, univariable and multivariable competing risks regression analyses were conducted [24] because smoking is an established risk factor for common health problems that increase risk of death. The cumulative incidence was estimated; patients who died without experiencing the event of interest were treated as a competing event. The Gray test was used to determine differences in cumulative incidence function between groups [24]. In multivariable analyses, potential interactions were tested using the likelihood ratio test. All reported $p$ values are two sided, and statistical significance was set at $p<0.05$. Statistical tests were performed with SPSS v.20 (SPSS, IBM Corp., Armonk, NY, USA) and R v.2.15.0 (R Foundation for Statistical Computing, Vienna, Austria).

\section{Results}

\subsection{Association of smoking with clinicopathologic characteristics}

Of 864 patients, 244 (28.2\%) never smoked, 297 (34.4\%) were former smokers, and 323 (37.4\%) were current smokers. Most of the former and current smokers smoked <20 CPD with a duration >20 yr. Among ever smokers, 87 (10.1\%), 331 (38.3\%), and 202 (23.4\%) were light-short-term, moderate, and heavy-long-term smokers, respectively.

Table 1 shows the descriptive characteristics of the study cohort. Current smokers were younger than former and never smokers (69 vs 71 and $70 \mathrm{yr}$, respectively; $p=0.025$ ). Compared with never smokers, former and current smokers had higher tumor stages ( $p<$ $0.001)$ and grades $(p=0.009)$, more lymph node metastasis $(p<0.001)$, and more tumor necrosis $(p=0.048)$. On logistic regression analyses, compared with never smokers, smoking status, quantity, duration, and cumulative exposure were all significantly associated with muscle-invasive disease and/or lymph node metastasis ( $p$ values $\$ 0.004$ ) and nonorgan-confined disease and/or lymph node metastasis ( $p$ values $₫ \mathbf{\$} .022$; Table 2). The odds of having advanced disease were highest among current smokers, as well as those smoking $\geq 20$ CPD or with heavy-long-term cumulative exposure (Table 2). In the subgroup analyses of former smokers, smoking $\geq 20 \mathrm{CPD}$ or having a heavy-long-term cumulative exposure was significantly associated with muscle-invasive disease and/or lymph node metastasis and non-organ-confined disease and/or lymph node metastasis ( $p$ values $\unlhd$ D.05). In subgroup analyses of current smokers, each stratification of smoking quantity, duration, and cumulative exposure was significantly associated with both end points compared with never smokers ( $p$ values $\mathbf{\$} 0.015$ ).

\subsection{Association of smoking with survival outcomes}

Median follow-up of patients alive at censorship was 50 mo (interquartile range: 23-90). A total of 605 patients $(70 \%)$ had $>24$ mo of follow-up available. During follow-up, disease recurred in 273 patients (31.6\%), $359(41.6 \%)$ died of any cause, and $220(25.5 \%)$ died of UTUC. Actuarial recurrence-free survival estimates at 2, 5, and $10 \mathrm{yr}$ after RNU were $69 \pm$ 
$2 \%$ (standard error), $64 \pm 2 \%$, and $60 \pm 2 \%$, respectively. Actuarial cancer-specific survival estimates at 2, 5, and $10 \mathrm{yr}$ after RNU were $79 \pm 2 \%, 69 \pm 2 \%$, and $62 \pm 2 \%$, respectively.

Current and former smokers had a higher risk of disease recurrence ( $p$ values $\$ 0.016$ ) and cancer-specific mortality compared with never smokers ( $p$ values $₫ 0.005$; Fig. 1 ). In addition, current smokers had a higher risk of disease recurrence $(p=0.017)$ compared with former smokers. Light-short-term, moderate, and heavy-long-term smokers had higher risks of disease recurrence compared with never smokers ( $p$ values $\unlhd \mathbf{0} .033$; Fig. 2). Heavy-longterm smokers had a higher risk of cancer-specific mortality compared with never smokers $(p$ value $<0.001$ ), as well as a higher risk for both end points compared with moderate smokers ( $p$ values $\mathbf{}$ ).031). There was no difference between light-short-term and moderate smokers and, interestingly, light-short-term and heavy-long-term smokers for both end points (Fig. 2).

In the subgroup of former smokers, heavy-long-term smokers had a significantly higher risk for both end points compared with never, light-short-term, and moderate smokers ( $p$ values $\$$ ).034), who in turn were not different from each other $(p>0.05)$. In contrast, in the subgroup of current smokers, light-short-term, moderate, and heavy-long-term smokers had a significantly higher risk for both end points compared with never smokers $(p \leq 0.011)$. Light-short-term, moderate, and heavy-long-term smokers were not significantly different from each other in this subgroup. When analyzing patients according to smoking quantity and duration separately, those who smoked $\geq 20 \mathrm{CPD}$ or $\geq 20 \mathrm{yr}$ had a higher risk for both

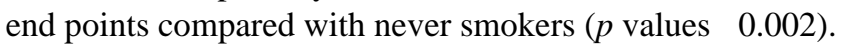

On multivariable analyses that adjusted for the effects of standard clinicopathologic parameters, current smoking status was associated with disease recurrence (hazard ratio [HR]):1.66; 95\% confidence interval [CI], 1.18-2.34; $p=0.003)$ and cancer-specific mortality (HR: 1.54; 95\% CI, 1.00-2.07; $p=0.05$; Table 3a). In addition, former smokers had a significantly higher risk of cancer-specific mortality (HR: 1.48; 95\% CI, 1.01-2.17; $p$ $=0.044)$. Compared with never smokers, smokers with $\geq 20 \mathrm{CPD}, \geq 20 \mathrm{yr}$ of smoking, or heavy-long-term smokers had a significantly higher risk of both disease recurrence ( $p$ values $₫ .01)$ and cancer-specific mortality $(p \leq 0.02)$. In addition, smokers with $\leq 19 \mathrm{CPD}$ and moderate smokers had a higher risk of disease recurrence compared with never smokers ( $p \leq$ 0.044; Table 3b).

\subsection{Effect of smoking cessation on clinicopathologic characteristics and outcomes}

In 297 former smokers, 204 (68.7\%) quit smoking <10 yr (recent former smokers) and 93 $(31.3 \%)>10 \mathrm{yr}$ (distant former smokers) prior to RNU. Compared with never smokers, current and recent former smokers had increased risks of muscle-invasive, non-organconfined disease, or lymph node metastasis on multivariable analyses that adjusted for the effects of age, gender, and tumor location ( $p$ values $<0.001$; Table 2$)$.

The cumulative incidences of disease recurrence and cancer-specific mortality according to smoking status and time since cessation are shown in Figure 3. There was no difference in outcomes for both end points between current and recent former smokers as well as between never and distant former smokers ( $p$ values $>0.05$ ). Current smokers and recent former smokers had a significantly higher risk of disease recurrence and cancer-specific mortality than never smokers ( $p$ values $\$ \mathbf{0 . 0 1 1}$ ). Smoking cessation was not associated with disease recurrence or cancer-specific mortality on multivariable analyses ( $p$ values $>0.05$ ).

To elaborate the association of time from cessation and cumulative smoking intensity, we stratified former smokers by both variables. Of the 297 former smokers, all light smokers (66 [22.2\%]) were distant former smokers, all moderate smokers (126 [42.4\%]) were recent 
former smokers, and there were 105 heavy smokers (35.4\%). Of these heavy smokers, 27 $(9.1 \%)$ quit $>10 \mathrm{yr}$ and $78(26.3 \%)<9.9 \mathrm{yr}$ prior to RNU. Using never smokers as a reference group in univariable Cox regression analyses, light former smokers had a reduced risk of disease recurrence (HR: 0.67) and cancer-specific mortality (HR: 0.55). For both end points, these odds increased for moderate smokers (HRs: 1.17 and 1.18, respectively), distant former heavy smokers (HRs: 1.92 and 2.69, respectively), and recent former heavy smokers (HRs: 2.19 and 2.69, respectively).

\section{Discussion}

Almost two thirds of patients in our study were current or former smokers, underscoring the close association of cigarette smoking and the development of UTUC [5]. Cigarette smoking is an established risk factor for the development of UC of both the upper and lower urinary tract $[2,23]$. There is little knowledge regarding the effect of smoking on UTUC progression and outcomes, however. A better understanding of these associations could offer insights into the natural history of UTUC, as well as improve patient counseling and possibly outcomes through modification of smoking habits.

We found that current smokers were at a significantly higher risk of advanced disease stages and lymph node metastasis compared with never smokers. Current smokers had a higher risk of disease recurrence as well as current and former smokers for cancer-specific mortality. The odds of having advanced disease and the likelihood of disease recurrence and cancerspecific mortality increased progressively from never to former to current smokers. Similar findings have previously been reported in other urologic malignancies such as prostate and renal cell cancer [10,25]. However, the magnitude of the odds was higher for standard clinicopathologic features than for smoking, which is similar to previous findings in UCB [26]. The failure to find a statistical significant association between former smoking status and disease recurrence in multivariable analyses might be explained due to the strength of the covariables (ie, pathologic stage, grade, and lymph node status), which are established predictors of outcomes, as confirmed in our study $[6,8,27]$. In addition, controlling for intermediate variables (such as clinicopathologic variables) can result in overadjustment that usually biases estimates toward the null [28].

We found a dose relationship between cumulative smoking exposure and risk of developing advanced disease stages and poor outcomes. Although never smokers had the most favorable outcomes, heavy-long-term smokers had the worst. Heavy-long-term cumulative smoking exposure was an independent risk factor for features of biologically aggressive UTUC (ie, advanced disease stages and lymph node metastasis) as well as disease recurrence and cancer-specific mortality after adjusting for the effects of established features. Indeed, the odds for each end point were highest in heavy-long-term smokers. In addition, patients with the highest smoking quantity and duration presented with the worst outcomes. Although there is no comparable study in UTUC, similar findings have been reported in UCB [10,12]. Two large studies found that stage and grade increased according to smoking status and with increasing quantity of smoking. Although the exact mechanisms of smoking-induced urothelial carcinogenesis remain unknown, accumulating evidence suggests that dose escalation and longer duration might increase not only the risk of development of UC but also its aggressiveness and the risk of progression to advanced disease [2,11,12,23].

We found that smoking cessation $>10 \mathrm{yr}$ seemed to mitigate the unfavorable effects of smoking in UTUC patients. Distant former smokers had less advanced disease stages, fewer disease recurrences, and lower cancer-specific mortality compared with recent former or current smokers. This beneficial effect in patients with long-term smoking cessation may be due to minor field damage effects or better retained repair mechanisms $[21,26]$. Although 
our results confirmed the need of long-term smoking cessation to reduce the effect of smoking in UC [2,21,26], this effect was not present in all former smokers. Smoking intensity seems to have an important impact on outcomes; a combination of smoking with other inherent/genetic, environmental, behavioral, or lifestyle factors that our study could not adjust for might be another explanation [29].

Our study has several limitations. First and foremost are limitations inherent to the retrospective and multi-institutional nature of the study including surgeons' numbers, preferences, experience, and caseload, as well as surgical techniques. Absence of lymphadenectomy in a significant number of patients as well as differences in the lymph node count and anatomic template of lymphadenectomy might have influenced our results. Another limitation includes possible interobserver variability between pathologists; a central pathology review was not performed. However, all surgeons and pathologists operated at centers dedicated to the management of UTUC. Comorbidities might have influenced the decision making regarding surgical therapy, introducing a selection bias. Another bias might be the exclusion of tobacco products other than cigarettes (eg, cigars, pipes, tobacco chewing) and different forms of tobacco exposure (eg, secondhand smoking, occupational exposure). We could also not adjust our analyses for different types of tobacco and its constituents. Finally, smoking history was self-reported and therefore subject to recall bias.

\section{Conclusions}

Cigarette smoking is significantly associated with advanced disease stages, lymph node metastasis, disease recurrence, and cancer-specific mortality in patients treated with RNU for UTUC. Current and heavy-long-term smokers have the highest risk for poor outcomes. Smoking cessation $>10 \mathrm{yr}$ prior to surgery seems to reduce the unfavorable effects of smoking on outcomes. Although our results need to be confirmed in a robust prospective study, these findings should further help urologists and general health care practitioners to counsel their patients regarding the benefits of smoking cessation and prevention programs.

\section{Supplementary Material}

Refer to Web version on PubMed Central for supplementary material.

\section{Acknowledgments}

Funding/Support and role of the sponsor: None.

The authors thank all members of the Upper Tract Urothelial Carcinoma Collaboration (UTUCC): Patrick J. Bastian, Karim Bensalah, Evi Comploj, Mario Fernández, Vincenzo Ficarra, Wareef Kabbani, Wassim Kassouf, Eiji Kikuchi, Theresa M. Koppie, Juan I. Martinez-Salamanca, Surena F. Matin, Francesco Montorsi, Casey K. Ng, Giacomo Novara, Mototsuga Oya, Jean-Jacques Patard, Mesut Remzi, Marco Roscigno, Christian Seitz, Philipp Ströbel, Stefan Tritschler, Matthias Waldert, Thomas J. Walton, Alon Weizer, J. Stuart Wolf, and Richard Zigeuner

\section{References}

1. Roupret M, Zigeuner R, Palou J, et al. European guidelines for the diagnosis and management of upper urinary tract urothelial cell carcinomas: 2011 update. Eur Urol. 2011; 59:584-94. [PubMed: 21269756]

2. Colin P, Koenig P, Ouzzane A, et al. Environmental factors involved in carcinogenesis of urothelial cell carcinomas of the upper urinary tract. BJU Int. 2009; 104:1436-40. [PubMed: 19689473]

3. Pommer W, Bronder E, Klimpel A, et al. Urothelial cancer at different tumour sites: role of smoking and habitual intake of analgesics and laxatives. Results of the Berlin Urothelial Cancer Study. Nephrol Dial Transplant. 1999; 14:2892-7. [PubMed: 10570093] 
4. Siegel R, Naishadham D, Jemal A. Cancer statistics, 2012. CA Cancer J Clin. 2012; 62:10-29. [PubMed: 22237781]

5. McLaughlin JK, Silverman DT, Hsing AW, et al. Cigarette smoking and cancers of the renal pelvis and ureter. Cancer Res. 1992; 52:254-7. [PubMed: 1728398]

6. Margulis V, Shariat SF, Matin SF, et al. Outcomes of radical nephroureterectomy: a series from the Upper Tract Urothelial Carcinoma Collaboration. Cancer. 2009; 115:1224-33. [PubMed: 19156917]

7. Chromecki TF, Bensalah K, Remzi M, et al. Prognostic factors for upper urinary tract urothelial carcinoma. Nat Rev Urol. 2011; 8:440-7. [PubMed: 21727942]

8. Kikuchi E, Margulis V, Karakiewicz P, et al. Lymphovascular invasion predicts clinical outcomes in patients with node-negative upper tract urothelial carcinoma. J Clin Oncol. 2009; 27:12-8.

9. Fleshner N, Garland J, Moadel A, et al. Influence of smoking status on the disease-related outcomes of patients with tobacco-associated superficial transitional cell carcinoma of the bladder. Cancer. 1999; 86:2337-45. [PubMed: 10590376]

10. Joshu CE, Mondul AM, Meinhold CL, et al. Cigarette smoking and prostate cancer recurrence after prostatectomy. J Natl Cancer Inst. 2011; 103:835-8. [PubMed: 21498781]

11. Lammers RJ, Witjes WP, Hendricksen K, et al. Smoking status is a risk factor for recurrence after transurethral resection of non-muscle-invasive bladder cancer. Eur Urol. 2011; 60:713-20. [PubMed: 21794974]

12. Sturgeon SR, Hartge P, Silverman DT, et al. Associations between bladder cancer risk factors and tumor stage and grade at diagnosis. Epidemiology. 1994; 5:218-25. [PubMed: 8172997]

13. Thompson IM, Peek M, Rodriguez FR. The impact of cigarette smoking on stage, grade and number of recurrences of transitional cell carcinoma of the bladder. J Urol. 1987; 137:401-3. [PubMed: 3820367]

14. Chromecki TF, Cha EK, Fajkovic H, et al. The impact of tumor multifocality on outcomes in patients treated with radical nephroureterectomy. Eur Urol. 2012; 61:245-53. [PubMed: 21975249]

15. Roscigno M, Shariat SF, Margulis V, et al. The extent of lymphadenectomy seems to be associated with better survival in patients with nonmetastatic upper-tract urothelial carcinoma: how many lymph nodes should be removed? Eur Urol. 2009; 56:512-8. [PubMed: 19559518]

16. Edge, SB.; Byrd, DR.; Compton, CC., et al. AJCC staging manual. 7. New York, NY: Springer; 2010.

17. Eble, JN.; Sauter, G.; Epstein, JI.; Sesterhenn, IA. Pathology and genetics of tumours of the urinary system and male genital organs. Lyon, France: IARCC Press; 2004. World Health Organization classification of tumours.

18. Remzi M, Haitel A, Margulis V, et al. Tumour architecture is an independent predictor of outcomes after nephroureterectomy: a multi-institutional analysis of 1363 patients. BJU Int. 2009; 103:307-11. [PubMed: 18990163]

19. Zigeuner R, Shariat SF, Margulis V, et al. Tumour necrosis is an indicator of aggressive biology in patients with urothelial carcinoma of the upper urinary tract. Eur Urol. 2010; 57:575-81. [PubMed: 19959276]

20. Rink M, Fajkovic H, Cha EK, et al. Death certificates are valid for the determination of cause of death in patients with upper and lower tract urothelial carcinoma. Eur Urol. 2012; 61:854-5. [PubMed: 22226583]

21. Chen CH, Shun CT, Huang KH, et al. Stopping smoking might reduce tumour recurrence in nonmuscle-invasive bladder cancer. BJU Int. 2007; 100:281-6. discussion 286. [PubMed: 17419696]

22. Freedman ND, Silverman DT, Hollenbeck AR, Schatzkin A, Abnet CC. Association between smoking and risk of bladder cancer among men and women. JAMA. 2011; 306:737-45. [PubMed: 21846855]

23. Jiang X, Castelao JE, Yuan JM, et al. Cigarette smoking and subtypes of bladder cancer. Int J Cancer. 2012; 130:896-901. [PubMed: 21412765]

24. Kim HT. Cumulative incidence in competing risks data and competing risks regression analysis. Clin Cancer Res. 2007; 13:559-65. [PubMed: 17255278] 
25. Kroeger N, Klatte T, Birkhauser FD, et al. Smoking negatively impacts renal cell carcinoma overall and cancer-specific survival. Cancer. 2012; 118:1795-802. [PubMed: 21997347]

26. Aveyard P, Adab P, Cheng KK, et al. Does smoking status influence the prognosis of bladder cancer? A systematic review. BJU Int. 2002; 90:228-39. [PubMed: 12133057]

27. Novara G, De Marco V, Gottardo F, et al. Independent predictors of cancer-specific survival in transitional cell carcinoma of the upper urinary tract: multi-institutional dataset from 3 European centers. Cancer. 2007; 110:1715-22. [PubMed: 17724728]

28. Schisterman EF, Cole SR, Platt RW. Overadjustment bias and unnecessary adjustment in epidemiologic studies. Epidemiology. 2009; 20:488-95. [PubMed: 19525685]

29. Ferrucci L, Izmirlian G, Leveille S, et al. Smoking, physical activity, and active life expectancy. Am J Epidemiol. 1999; 149:645-53. [PubMed: 10192312] 


\section{Take-home message}

Current, heavy, and long-term cigarette smoking at the time of radical nephroureterectomy for treatment of upper tract urothelial carcinoma is significantly associated with advanced disease and poor outcomes. These findings should strongly encourage urologists and general health care practitioners to support smoking cessation programs 


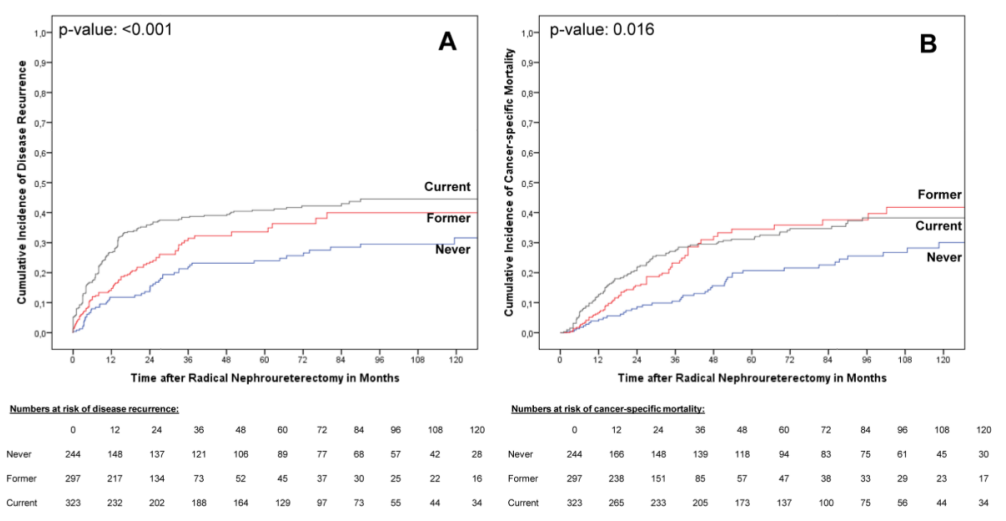

Fig. 1.

Estimates of (a) disease recurrence and (b) cancer-specific mortality incidences according to cigarette smoking status in 864 upper tract urothelial carcinoma patients treated with radical nephroureterectomy. The competing event groups are not displayed in all figures. 

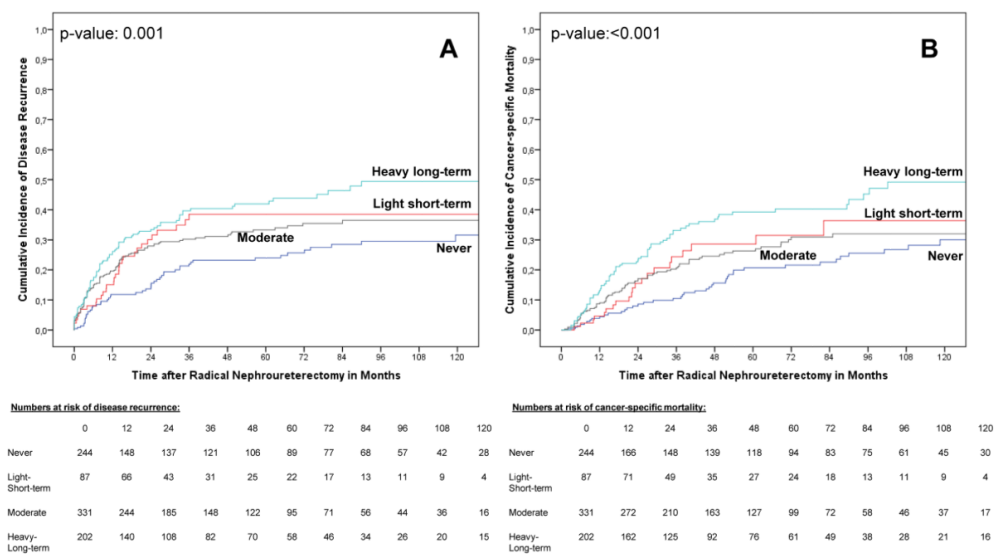

Fig. 2.

Estimates of (a) disease recurrence and (b) cancer-specific mortality incidences according to the cumulative smoking intensity in 864 upper tract urothelial carcinoma patients treated with radical nephroureterectomy. 

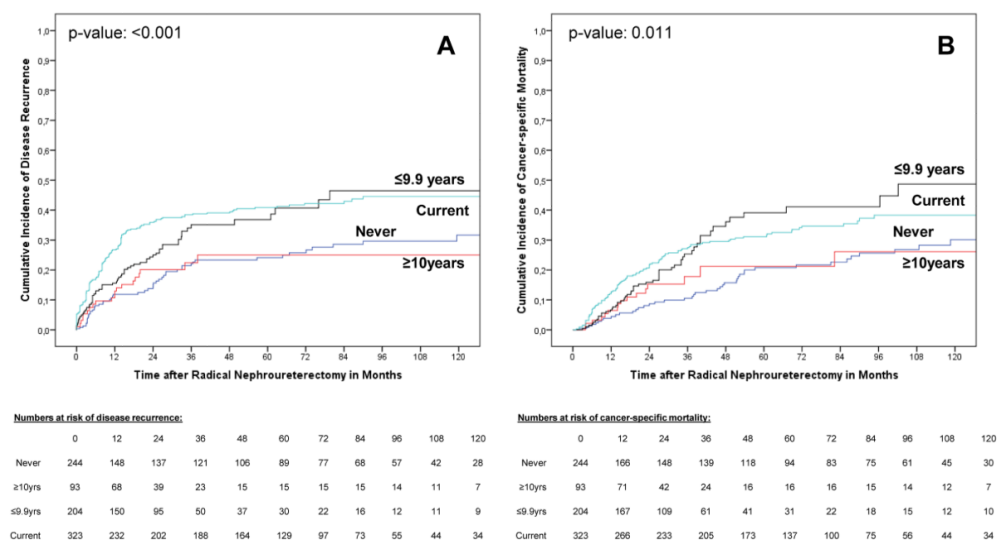

Fig. 3.

Estimates of (a) disease recurrence and (b) cancer-specific mortality incidences in 864 upper tract urothelial carcinoma patients treated with radical nephroureterectomy according to smoking status with consideration of time since cessation in former smokers. 


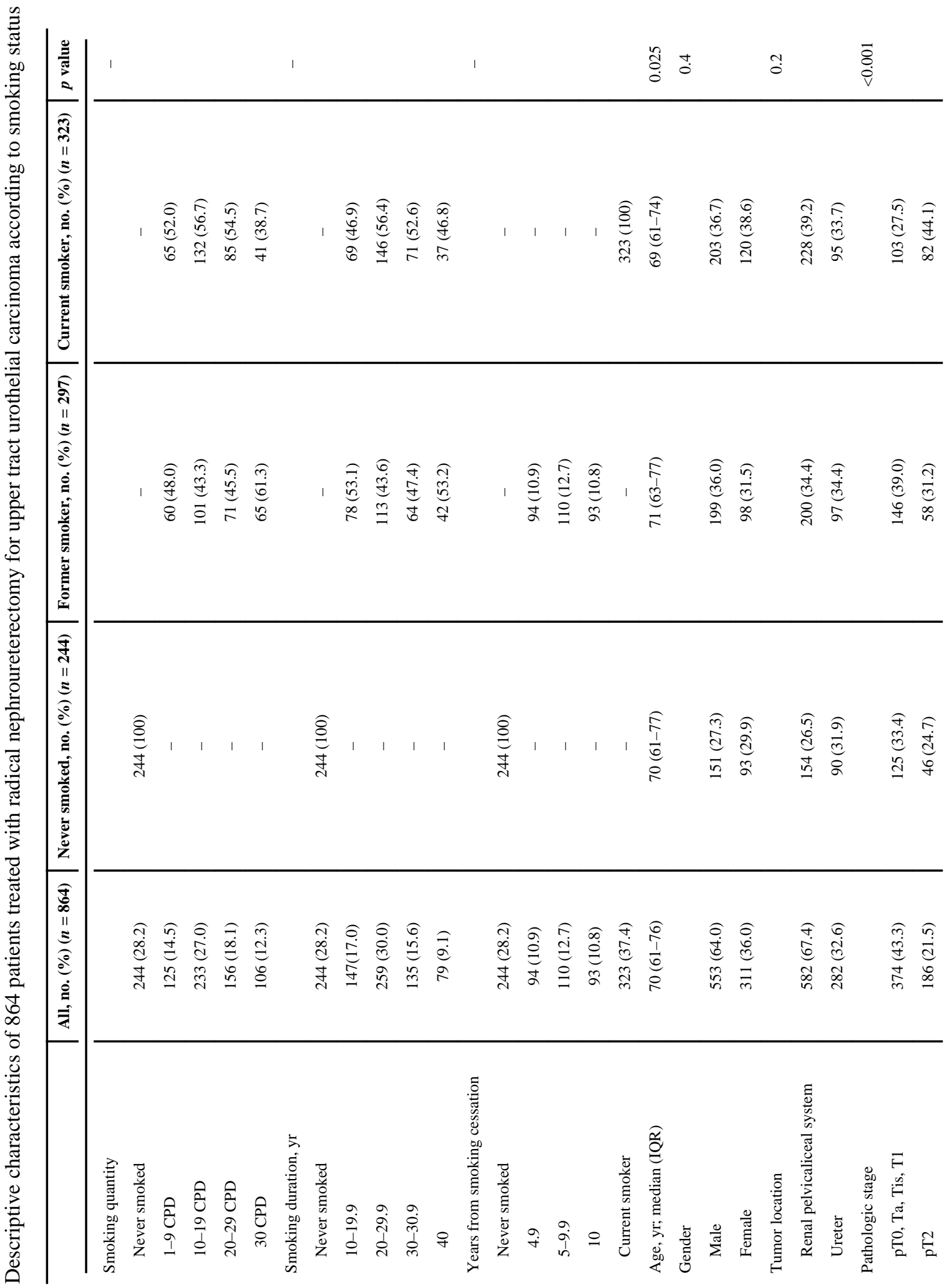


Rink et al.

Page 16

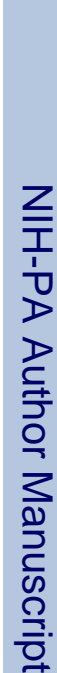

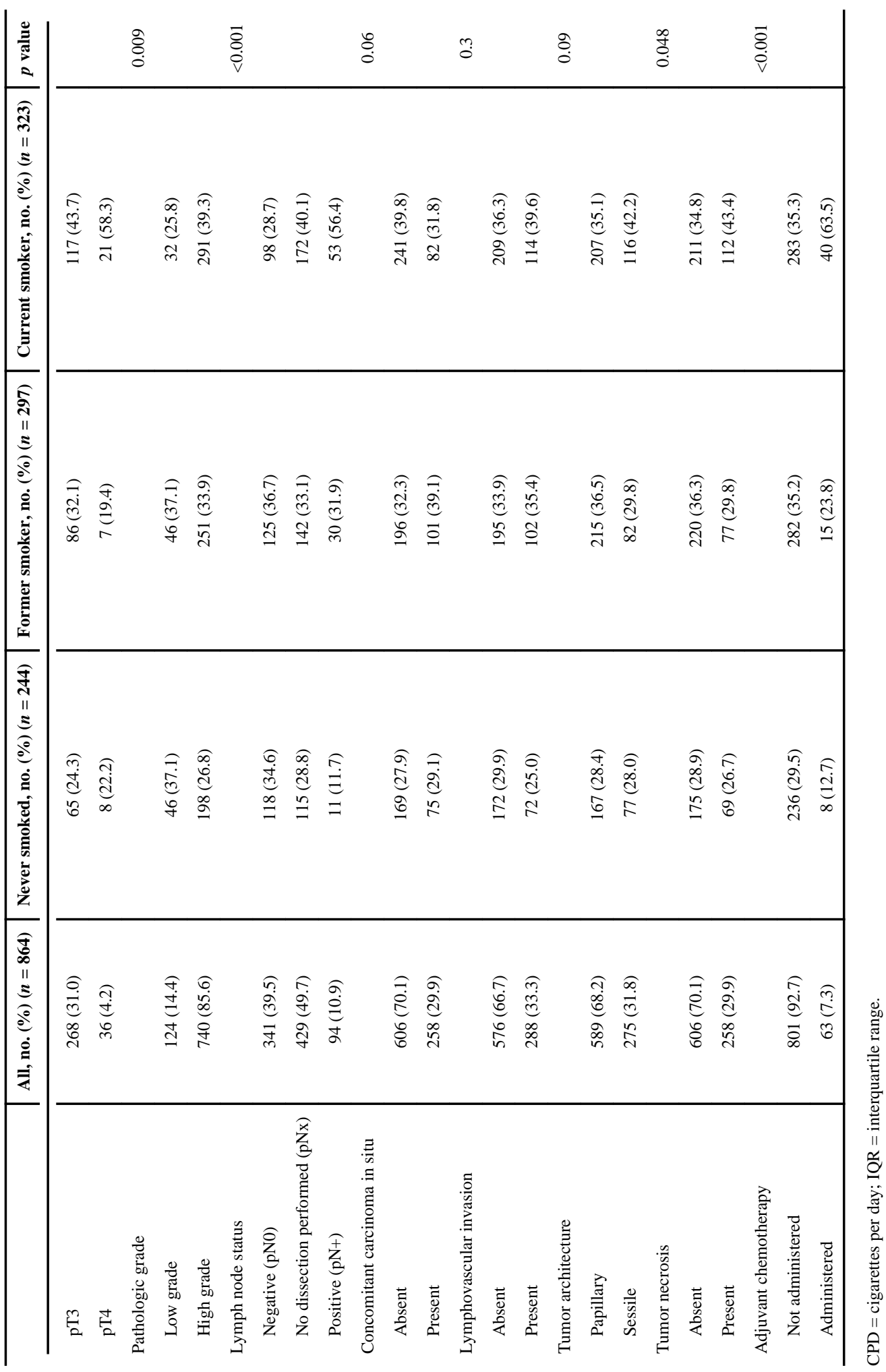

Eur Urol. Author manuscript; available in PMC 2014 June 01. 


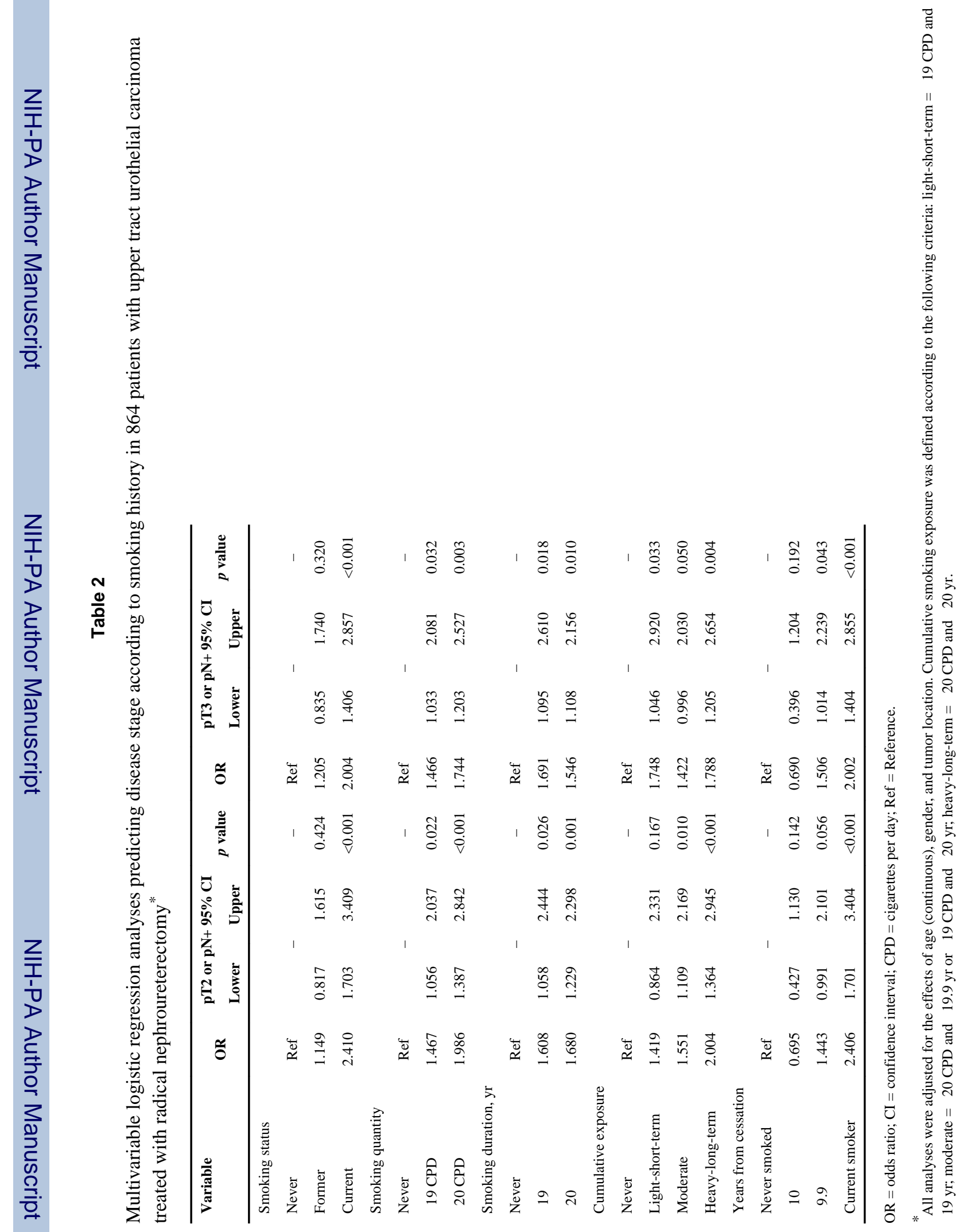




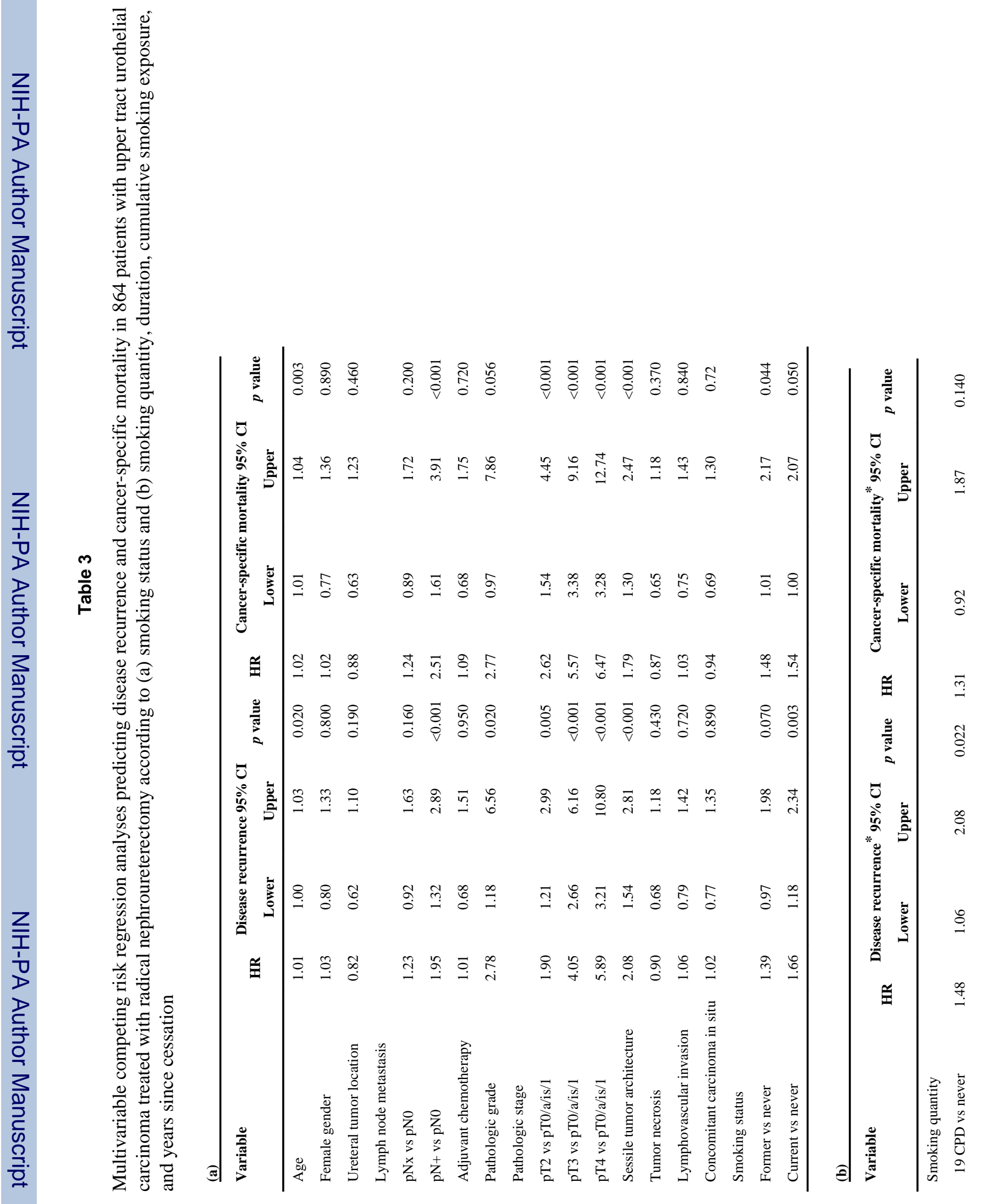




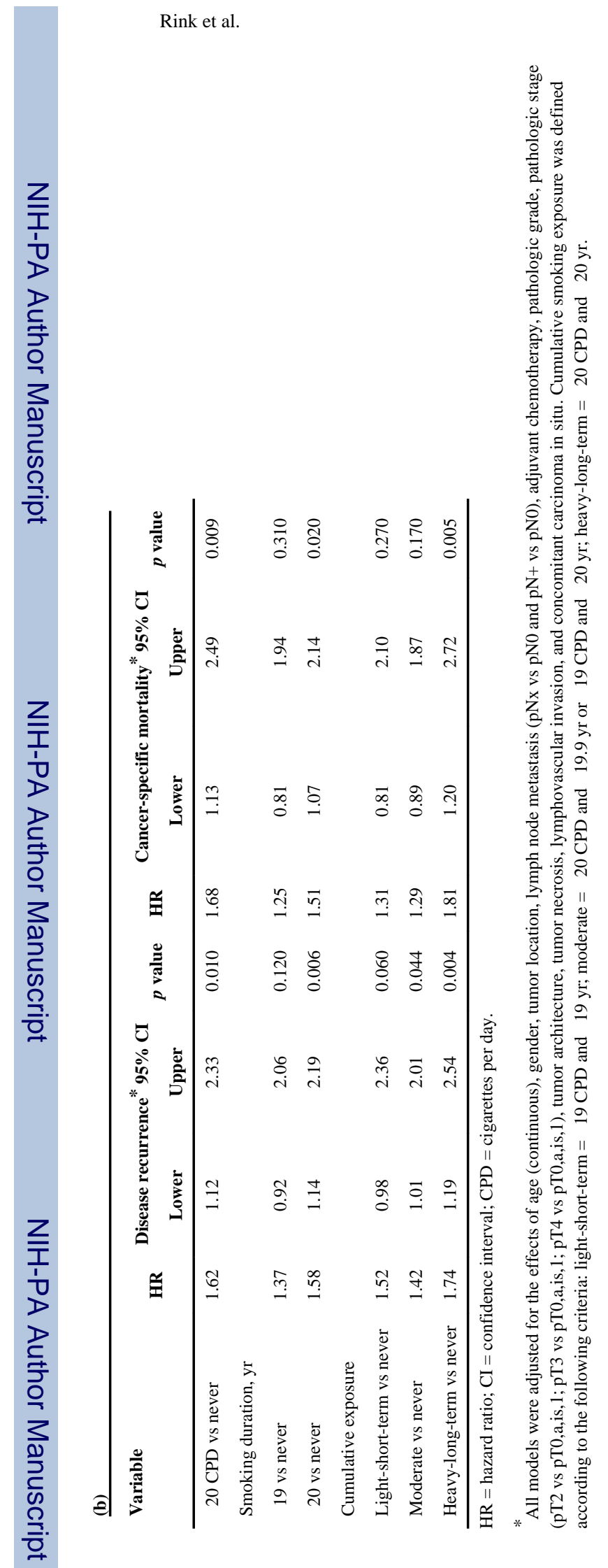

Eur Urol. Author manuscript; available in PMC 2014 June 01. 